\title{
Construcciones discursivas sobre el sujeto de la educación en la política educativa aprender
}

\author{
Construções discursivas sobre o sujeito da \\ educação na política educacional aprender \\ Discursive constructions on the subjects of \\ education in the aprender educational policy
}

\author{
CAMILA FALKIN \\ Orcid Id: http://orcid.org/0000-0003-0933-8545 \\ Universidad de la República, Uruguai \\ NALÚ FARENZENA \\ Orcid Id: ttp://orcid.org/0000-0003-2582-5925 \\ Universidade Federal do Rio Grande do Sul
}

\begin{abstract}
Resumen: El artículo trata de la política Atención Prioritaria en Entornos con Dificultades Estructurales Relativas (APRENDER), de la educación primaria uruguaya. Se discuten los sentidos construidos sobre el sujeto de la educación en una política focalizada en niños que viven en contextos de pobreza, partiendo de la perspectiva del Análisis Político del Discurso y de un corpus conformado por documentos oficiales y entrevistas a técnicos. Se identifica una tensión entre una concepción que subraya las posibilidades educativas de los sujetos y otra que, tanto en los discursos de los años 90 como en los de la era progresista, los visualiza como seres carentes.
\end{abstract}

Palabras clave: Políticas públicas de educación; Educación y desigualdad; Análisis Político del Discurso; Sujeto de la educación; APRENDER.

Resumo: $O$ artigo trata da politica Atención Prioritaria en Entornos con Dificultades Estructurales Relativas (APRENDER), da educação primária uruguaia. Discutemse sentidos construídos sobre o sujeito da educação numa política focalizada em crianças de contextos de pobreza, tendo como principal suporte a Análise Política do Discurso e como corpus documentos oficiais e depoimentos de gestores. Identifica-se uma tensão entre uma concepção que salienta as possibilidades educativas dos sujeitos e outra que, nos discursos dos anos 90 assim como nos da era progressista, os visualiza como seres carentes.

Palavras-chave: Políticas públicas de educaşão; Educação e desigualdade; Análise Politica do Discurso; Sujeito da educação; APRENDER. 
Abstract: The paper addresses the Uruguayan primary education Atencion Prioritaria en Entornos con Dificultades Estructurales Relativas (APRENDER) policy. The article discusses constructed meanings on the subjects of education in a policy focused on children living in impoverished contexts. It is based on the Political Analysis of Discourse and its corpus is formed by official documents and accounts from managers. Tension is identified between a conception that emphasizes the subjects' educational possibilities and another that, both in the discourses of the 1990s and in those of the progressive era, visualizes them as beings in need.

Keywords: Education public policies; Education and inequality; Political Analysis of Discourse; Subjects of education; APRENDER.

\section{INTRODUCCIÓN}

Las relaciones entre la educación escolar y la desigualdad social han sido un objeto privilegiado de un sinnúmero de discusiones, investigaciones y disputas políticas. En ese marco, y en función de la perspectiva asumida, con frecuencia se le ha atribuido a la escuela una importancia central en la construcción de una sociedad igualitaria y democrática o en la reproducción de un modelo social injusto y desigual. El presente artículo incorpora dichas preocupaciones, pero no pretende dilucidar de un modo apriorístico, unívoco y definitivo los sentidos que asumen las relaciones entre escolarización y desigualdad, sino analizar cómo estos sentidos son construidos, de manera contingente y provisoria, en el marco de una política educativa implementada en Uruguay.

Se presenta aquí, con base en una disertación de maestría aprobada recientemente, un análisis del programa Atención Prioritaria en Entornos con Dificultades Estructurales Relativas (APRENDER), política educativa focalizada en los sectores más pobres de la población uruguaya. Con relación a ese programa, nos enfocaremos particularmente en las concepciones sobre el sujeto de la educación que lo atraviesan y constituyen. Para el abordaje de esa temática, se recuperan aportes teóricos, analíticos e interpretativos del Análisis Político del Discurso (APD), perspectiva que tiene su procedencia en la línea de pensamiento desarrollada por Ernesto Laclau y Chantal Mouffe.

El programa APRENDER se implementa en la enseñanza primaria en Uruguay -nivel educativo equivalente a los seis años iniciales del "ensino fundamental” brasileño- desde el año 2011 y constituye una reformulación de una política anterior, las denominadas escuelas de Contexto Sociocultural Crítico, creadas en el año 1999. Esta intervención estatal puede ser considerada como una articulación de diversos programas y proyectos que, en su conjunto, responden más a una intención de dotar de mayores recursos a algunas escuelas que a una propuesta pedagógica coherente. El programa APRENDER agrupa a una serie de acciones con intencionalidades diferentes, pero con el rasgo común de estar 
focalizadas en las escuelas a las que acuden los niños que viven en situación de pobreza. En la actualidad, constituye la principal política focalizada de enseñanza primaria, abarcando en el 2019 al 29\% de los escolares matriculados en la educación pública común. Importa aclarar que la educación pública en Uruguay es ampliamente mayoritaria, las escuelas públicas de educación común recibieron en dicho año al 83\% del total de la matrícula nacional en este nivel educativo.

Ese programa representa la etapa más reciente de un proceso de larga data de clasificación de los sujetos y de las instituciones educativas en función de su vinculación con contextos de pobreza. Los inicios de ese proceso se remontan a la restauración democrática del año 1985 y se vinculan a la instauración de una serie de reformas neoliberales, que se consolidaron en el país en la década de los noventa. Esas reformas provocaron efectos duraderos en la estructura social y en el terreno educativo, a pesar de haber sido implementadas en Uruguay con menor profundidad que en otros países del continente. El despliegue de dichas reformas condujo a una reconfiguración de la modalidad de intervención estatal, de modo tal que las políticas sociales de integración, universales y homogeneizantes, cedieron lugar a las políticas de inserción (CASTEL, 1997), compensatorias y focalizadas. Eso supuso la demarcación de ciertas zonas y sectores de la población y su identificación como focos prioritarios de la intervención del Estado. En el caso de las escuelas, ello implicó la tipificación de algunas de ellas como de "requerimiento prioritario" o, posteriormente, como de "contexto sociocultural crítico", entre otras denominaciones que se han empleado.

En el campo educativo, ese proceso clasificatorio se vio reforzado con el desarrollo, entre los años 1995 y 2000, de una reforma educativa que, si bien operó en el marco de la agenda internacional neoliberal, constituyó una experiencia relativamente heterodoxa. Dicho proceso reformista tuvo una importancia crucial en la construcción de un discurso pedagógico, ligado al significante equidad, que aún hoy permea fuertemente el imaginario social y que posee una relación directa con la política que aquí se aborda. En términos generales, la equidad fue concebida como la "[...] compensación de las desventajas, mediante el trato desigual a quienes son socialmente desiguales” (DUHAU, 2001, p. 313). Ese significante desplazó a la noción de igualdad, uno de los ejes sobre los que se estructuró en Uruguay, a partir del último tercio del siglo XIX, el proyecto educativo moderno (BORDOLI, 2015a). Desde el discurso de la reforma educativa, como señala Martinis (2013), la educación fue concebida como una importante estrategia de lucha contra la pobreza, en relación con lo cual, las escuelas que recibían a los niños que vivían en esos contextos asumieron una multiplicidad de tareas vinculadas a la asistencia social. 
De este modo, desde de los años ochenta, y con mayor fuerza a partir de la reforma educativa de 1995, se han constituido diferentes tipos de escuela, en función de los contextos sociales de los alumnos y, durante algunos años, de sus resultados educativos, a partir de lo cual se ha definido la focalización de algunas políticas compensatorias. Ese proceso de clasificación de las escuelas y de los sujetos que asisten a ellas se ha profundizado y tecnificado desde entonces, se ha centrado exclusivamente en los contextos socioculturales de los estudiantes -dejando de lado cualquier indicador relativo a factores educativos- y ha ido de la mano con la naturalización de una construcción discursiva que asume a la pobreza como un obstáculo para el desarrollo de aprendizajes.

En el año 2005, el Frente Amplio, coalición política de centroizquierda, llega al gobierno nacional -en el que permanecería hasta febrero del 2020-, oponiéndose al proceso de reformas desplegado en la década anterior y proponiendo una mayor intervención estatal frente a las injusticias sociales. Con relación al campo educativo, se destaca en esta etapa del país un esfuerzo por construir a la educación como un derecho de carácter universal. La aprobación de la Ley General de Educación (URUGUAY, 2008), durante el primer período de gobierno de esta fuerza política, constituye un hito en este sentido, ya que establece su consideración como un derecho humano fundamental y un bien público y social. Frente a los procesos de fragmentación instalados en las décadas precedentes, se recupera entonces la posibilidad de nombrar a la educación en términos de lo universal y lo común (MARTINIS, 2015).

En ese contexto, las políticas de inserción, concebidas inicialmente como emergenciales, continúan, pero son dotadas de nuevos sentidos. Las políticas focalizadas, entre ellas las denominadas políticas de inclusión educativa, son entendidas entonces como un "puente" (BARÁIBAR, 2016) para el pasaje a la universalidad y a la integración social. Es decir, a pesar de su carácter focalizado, poseerían, según postulan algunos actores, una “[...] vocación igualitaria y universal [...]” (BORDOLI, 2012, p. 19). La tensión que surge entonces, y que constituye un aspecto nodal de la discusión relativa a las políticas educativas, es, como plantea Bordoli (2012), “[...] hasta qué medida el establecimiento y tratamiento del otro como un des-igual colabora en su re-inscripción en el nosotros colectivo o coadyuva en un incremento de su discriminación" (p. 23).

La categoría inclusión educativa toma fuerza en la política educativa uruguaya a partir del año 2008, en un intento por instalar una nueva perspectiva con respecto a la relación entre educación y pobreza, desplazando, parcialmente, al significante equidad. Esa categoría está atravesada por una fuerte polisemia, 
que da lugar a una tensión constante entre el carácter educativo y/o vinculado a la protección social de las propuestas que se construyen apoyadas en ella (FLOUS, 2015). A ese respecto, Martinis (2015) identifica una cierta continuidad entre los discursos reformistas de la década de los noventa y los más recientes discursos progresistas, así como una familiaridad entre los significados que se asocian a la noción de equidad y aquellos vinculados al significante inclusión. Esa continuidad discursiva refiere a la construcción de los sujetos que viven en contextos de pobreza como sujetos excluidos, excepcionales, que portarían un conjunto de carencias que dificultarían el desarrollo de la acción educativa y ameritarían intervenciones específicas. Dicha construcción entra en tensión, de acuerdo con el autor (2015), con la concepción de la educación como un derecho universal y con el reconocimiento de todos los miembros de una sociedad como dignos y aptos para ejercerlo.

Es sobre la base de este proceso y de esa tensión que se crean en el 2011 las escuelas APRENDER, política de inclusión educativa instaurada en el marco de gobiernos progresistas que impulsaron en el país procesos de democratización del sistema educativo. Consideramos que resulta pertinente analizar las construcciones discursivas que dotan de sentido a ese programa, cuyos antecedentes remiten a un contexto político y social sumamente diferente y a un conjunto de significaciones que, en una primera lectura, parecerían diferir de aquellas que el progresismo pretendía promover.

En ese marco, nos importa analizar cómo se significó desde el discurso pedagógico progresista el desarrollo de la acción educativa en contextos de desigualdad social. Particularmente, nos centraremos en las concepciones sobre los sujetos de la educación que atraviesan y constituyen a ese programa que se focaliza en niños que viven en contextos de pobreza. Nos preguntamos entonces, ¿qué sentidos se construyen desde el programa APRENDER con relación a los sujetos de la educación y sus relaciones con el saber?

Postulamos que esas construcciones discursivas tienen efectos cruciales en el desarrollo de políticas y prácticas pedagógicas en contextos de desigualdad social. Si desde las políticas educativas se naturalizan los procesos de desigualdad, difícilmente se planteen horizontes de igualdad. Si los maestros colocan en acción estas políticas desconfiando de las capacidades de aprender de los estudiantes, se resienten fuertemente las posibilidades de construir un discurso pedagógico igualitario. 
En el siguiente apartado presentaremos algunas herramientas conceptuales y el enfoque analítico desde el cual abordaremos esa cuestión, para luego centrarnos específicamente en el programa APRENDER, primero en sus rasgos generales y, seguidamente, en el análisis de los sentidos construidos con relación al sujeto de la educación.

\section{ANÁLISIS POLÍTICO DEL DISCURSO Y POLÍTICAS EDUCATIVAS}

Con base en la perspectiva del Análisis Político del Discurso, la noción de discurso que se pone en juego en este artículo incluye cualquier tipo de acto u objeto que involucre una relación de significación (BUENFIL, 1993). Discurso refiere aquí a una "[...] configuración significativa, que involucra acciones lingüísticas y extralingüísticas, que es constitutiva de lo social y cuyo carácter es relacional, diferencial, abierto, incompleto, precario y susceptible de ser trastocado por una 'exterioridad constitutiva"' (BUENFIL, 2011, p. 10).

Este marco analítico parte de un posicionamiento antiesencialista, que entiende que las identidades sociales carecen de esencias y son construidas discursivamente, mediante articulaciones abiertas y contingentes. Este enfoque no niega la existencia de los objetos, su soporte material no desaparece, pero afirma que estos objetos únicamente pueden tornarse inteligibles en el marco de un campo discursivo, a partir de su dimensión significante y política, de su inscripción en la vida social; se afirma el "[...] carácter histórico, contingente y construido del ser de los objetos [...]" (LACLAU; MOUFFE, 1993, p. 126, énfasis de los autores). En este sentido, las políticas y prácticas educativas solo pueden ser comprendidas por su inserción en una configuración significativa. Como sostiene Buenfil, “Todo objeto o práctica es significada de alguna manera al ser apropiada por los agentes sociales. Toda configuración social es discursiva en este sentido. Las prácticas educativas, qua prácticas sociales, son también discursivas" (1993, p. 5, énfasis de la autora). De acuerdo con esta autora, “[...] no hay prácticas educativas al margen de una estructuración de significaciones” (1993, p. 7-8).

Los discursos sobre los estudiantes, sobre la función de la escuela y las identidades docentes que se construyen no responden a una esencia, estable, fija y cerrada, que determina lo que la escuela, los estudiantes y los docentes son y hacen. Por el contrario, se entiende que se producen en relación, a partir de disputas, en determinadas condiciones históricas y mediante procesos de significación que incluyen algunos sentidos y excluyen otros y que poseen una dimensión política, en tanto involucran relaciones de poder, conflictos y antagonismos. 
Lo social es concebido desde ese enfoque como una construcción discursiva de significado, como el juego infinito de las diferencias y el intento, siempre inacabado, de limitar ese juego, de fijar algún orden e instituir la sociedad (LACLAU, 1993). Desde el APD, la sociedad, como objeto unitario e inteligible, es un imposible. Esta perspectiva postula el carácter abierto de todo discurso, la imposibilidad de un cierre, de una fijación absoluta de significados, y es esa ausencia de una sutura última, lo que habilita la posibilidad de la disputa por la necesaria fijación parcial de sentidos.

Partiendo desde ese enfoque, y asumiendo el carácter precario e históricamente contingente de toda articulación discursiva, nos proponemos encontrar algunas regularidades en los discursos que atraviesan al programa APRENDER, distinguir puntos discursivos privilegiados que estén fijando parcialmente sentidos en torno a los estudiantes y sus relaciones con el conocimiento. Conforme a la concepción de política educativa en la que se apoya este trabajo, buscaremos esas articulaciones discursivas en los documentos que instituyen y regulan el programa y nos aproximaremos también a la construcción de la política en la práctica, a la producción de sentidos en su proceso de implementación.

Siguiendo a autores como Ferraço y Carvalho (2012) y Ball, Maguire y Braun (2016), entendemos que no es posible trazar una separación tajante entre política y práctica. Desde esta concepción, las políticas son prácticas,

[...] são, necessariamente, práticas pessoais e coletivas dentro de um campo qualquer no qual há, sempre, lutas de posições diferentes e mesmo contrárias. Dessa maneira, as pessoas e os grupos, em suas ações, produzem políticas que, muitas vezes, não são visíveis aos que analisam "as políticas" (FERRAÇO; CARVALHO, 2012, p. 3).

En este sentido, las prácticas son también políticas, como plantean estos autores con relación al ámbito educativo, “[...] são políticas negociadas nas complexas redes cotidianas de saberes, fazeres e poderes das escolas e dos sistemas educacionais" (FERRAÇO; CARVALHO, 2012, p. 3).

En consonancia con ello, en este trabajo buscaremos recuperar las construcciones discursivas sobre los sujetos de la educación puestas a circular por los textos oficiales vinculados al programa APRENDER; y nos aproximaremos también a los sentidos construidos desde la red que permite colocar dichos textos en acción (BALL; MAGUIRE; BRAUN, 2016). Para ello, trabajaremos a partir de documentos macro de la política educativa nacional, de los documentos institucionales de este programa en particular y de los posicionamientos asumidos por tres actores con importantes cargos de gestión e incidencia cotidiana en su 
implementación, recogidos por medio de entrevistas semiestructuradas realizadas en el año 2019. No abordaremos en este artículo las posiciones que los docentes construyen en su trabajo diario, pero no pretendemos con ello subestimar su importancia crucial para la producción de la política educativa.

Habiendo esbozado ya el entramado teórico que guiará nuestro trabajo, nos centraremos ahora en el programa APRENDER y en las construcciones discursivas, con sus tensiones y contradicciones, que lo atraviesan y constituyen.

\section{LA FOCALIZACIÓN COMO PROPUESTA}

En las últimas décadas, se han desplegado en Uruguay múltiples programas educativos con relación a las poblaciones que viven en situación de pobreza, programas focalizados que, hace poco más de diez años, se han dado en llamar políticas de inclusión educativa. El programa APRENDER se inscribe en dicho marco y se centra en escuelas y jardines clasificados como quintil 1 y 2 del Índice de Contexto Sociocultural (ICSC).

Ese índice fue calculado por primera vez en el año 2005, luego de la llegada del Frente Amplio al gobierno nacional y del cambio de autoridades en la Administración Nacional de Educación Pública (ANEP), ente autónomo que tiene a su cargo la administración del sistema educativo público en varios niveles, entre otros, los de educación inicial y primaria. La ANEP realizó entonces un relevamiento de las características sociales y económicas de los hogares de los alumnos de estos dos niveles educativos, y con la información generada estableció nuevos criterios para la categorización de las escuelas y jardines de infantes, continuando con el proceso de clasificación iniciado en la década de los noventa. Esa categorización ha sido actualizada desde entonces de forma quinquenal.

El ICSC toma en consideración el nivel educativo de las madres de los niños, el nivel socioeconómico de sus hogares y, para el caso de los centros educativos urbanos, la inserción territorial de esos hogares y el vínculo de sus miembros con el sistema educativo formal (ANEP - CODICEN, 2012). En base a ese índice, se realiza un ordenamiento de las escuelas y se las agrupa en cinco quintiles o "niveles de contexto". "De esta forma, el Quintil 1 agrupa al 20\% de escuelas con mayor nivel de criticidad en el Índice, el Quintil 2 al siguiente 20\% y así sucesivamente, hasta el Quintil 5, conformado por el 20\% de escuelas con indicadores socioculturales más favorables" (ANEP - CODICEN, 2012, p. 22-23). Esa categorización se emplea para delimitar la población, objetivo de gran parte de las políticas focalizadas de enseñanza primaria, entre las cuales las escuelas APRENDER son las que tienen una mayor cobertura. En el año 2019 ese programa se implementó en el 25,8\% (237) de las 920 escuelas urbanas de 
educación común del país y abarcó a la amplia mayoría de los niños $(77,3 \%)$ matriculados en centros urbanos de educación común de los quintiles 1 y 2 .

Con relación a estos niños, según plantea un documento oficial (ANEP - CEIP, 2010a), el programa APRENDER se propone garantizar su acceso, permanencia y el sostenimiento de trayectorias escolares personalizadas y de calidad, reducir las tasas de repetición, abatir el ausentismo y mejorar sus niveles de aprendizaje. A su vez, esa propuesta procura transformar la cultura institucional de los centros educativos, a partir del desarrollo de un trabajo colaborativo e interdisciplinario y de la consolidación de colectivos docentes que generen proyectos educativos enmarcados en un plan institucional. Por último, ese programa fue creado también con el fin de fortalecer el vínculo de las escuelas con las familias y la comunidad, promoviendo la participación de los referentes adultos de los estudiantes y el trabajo conjunto de los actores institucionales y de la comunidad educativa.

Para alcanzar sus objetivos, APRENDER se apoya en otros programas y en diversos instrumentos de acción, a los que procura conferirles una cierta unicidad. La noción de "acción polivalente" (ANEP - CEIP, 2010b), empleada para definir al programa en la resolución que lo instituye, condice con esta característica de agrupar bajo una única propuesta a una serie de acciones que tienen en común su focalización en las escuelas de los quintiles más bajos de acuerdo al ICSC. Esa focalización es justificada desde las nociones de equidad, inclusión e igualdad de oportunidades, categorías recurrentes en los distintos documentos y en las entrevistas realizadas. De acuerdo con un documento institucional, el programa APRENDER “[...] procura articular diversas acciones centradas en contextos de pobreza en una propuesta pedagógica inclusiva y de calidad" (ANEP - CEIP, 2011, n.p.). Es posible apreciar la amplitud de este abanico de acciones en otro fragmento del mismo documento, el cual afirma que en estas instituciones,

[...] coinciden el Programa de Alimentación Escolar (PAE), el Programa de Maestros Comunitarios (PMC), una compensación salarial para los docentes que cumplen con una jornada mensual de trabajo en equipo en día sábado, un curso de Formación en Servicio, una partida especial de funcionamiento al inicio del año lectivo, la asignación priorizada de otros servicios de apoyo como el de equipos multidisciplinarios, y la reserva de financiamiento para proyectos de oportunidades de desarrollo escolar (ANEP - CEIP, 2011, n.p.).

El elemento que unifica a esta diversidad de acciones, y que representa la característica central del programa APRENDER, es su carácter focalizado. Ese programa se enmarca en un lineamiento de la política de educación inicial y primaria, establecido para el período 2016 - 2020, que postula que 
[...] es necesario continuar las líneas de política de focalización que atienden a los sectores más vulnerables de la sociedad. La atención de las escuelas en función de las características socioculturales de la comunidad a la que pertenecen sus alumnos, refleja la necesidad de entender el contexto para poder trabajar a partir de él [...] (ANEP - CEIP, 2016, p. 75).

Sostenemos que ese posicionamiento, fundamento del programa APRENDER, está directamente vinculado a una construcción discursiva que articula contextos de pobreza y obstáculos para el desarrollo de la acción educativa. Abordaremos ese punto en el siguiente apartado, que se centra en las concepciones sobre los sujetos de la educación que atraviesan y constituyen a ese programa.

\section{ENTRE LA POSIBILIDAD Y LOS “GRUPOS DE RIESGO”}

En las entrevistas realizadas y en los documentos analizados se señala recurrentemente la importancia de concebir a los sujetos de la educación desde la posibilidad, y desde la convicción de que todos son capaces de establecer relaciones significativas con el conocimiento. Partiendo desde esa posición, se plantea que a las instituciones educativas les caben responsabilidades para contribuir a desarrollar esas posibilidades de aprender que todos los niños tienen.

En este sentido, en un documento del programa se sostiene que

Las causas de la discontinuidad o interrupción de las trayectorias escolares no pueden atribuirse exclusivamente al contexto sociocultural de origen del niño. En este fenómeno inciden diversos factores, entre ellos, la oferta educativa de la escuela y la forma en que se atienden las demandas educativas de los niños (ANEP - CEIP, 2010a, n.p.).

De modo similar, un entrevistado, inspector referente del programa, luego de afirmar que todos los estudiantes pueden aprender, planteó que "Es una cuestión que va por la institución también, por el trabajo docente, hay otras herramientas, los chiquilines aprenden. Son estrategias, el maestro tiene estrategias didácticas para poder lograr el aprendizaje de todos los niños" (entrevista $\mathrm{N}^{\circ} 3$ ).

A su vez, la coordinadora nacional del programa APRENDER, en la entrevista que le fue realizada, hizo referencia a la tarea de la escuela y de los maestros de contribuir a que los niños puedan descubrir que son capaces de aprender. En palabras de la entrevistada: 
Si estará vinculado a esto de poder descubrir que es posible lograrlo, y del vínculo que establezcan con el maestro, eso es fundamental. [...] son niños en general bastante vulnerados, en sus derechos y en muchos aspectos de su vida, entonces poder revertir esto y demostrarles o permitirles descubrir, ayudarlos a descubrir que ellos son capaces de aprender, hace que rápidamente destraben estas dificultades que venían presentando y tengan buenos logros (entrevista $\mathrm{N}^{\circ} 1$ ).

Por su parte, otra entrevistada, la coordinadora nacional del Programa de Maestros Comunitarios (PMC), afirmó que “[...] cada niño es un ser distinto, ni mejor ni peor, y cada uno va a aprender de acuerdo a las posibilidades que se tengan" (entrevista $\mathrm{N}^{\mathrm{o}}$ 2). En este sentido, planteó la importancia de "[...] cambiar la perspectiva del niño con dificultad al niño, o a la familia, con posibilidad, con la oportunidad" (entrevista No 2) y consideró que "la mayor riqueza" del PMC es su aporte a este cambio. Esa mirada que coloca a los sujetos de la educación y a sus familias en el lugar de la posibilidad, así como el gesto de reconocer al otro en su singularidad, fue identificada por Bordoli (2015b) como pieza del entramado discursivo articulado en torno a ese programa.

El PMC fue creado en el primer año de gobierno del Frente Amplio, su trayectoria y objetivos difieren de los de APRENDER, pero ambos programas convergen en las mismas escuelas y, según afirma un documento institucional, "[...] la presencia del PMC es un componente fundamental en el diseño, la implementación, el desarrollo, el seguimiento y la síntesis de APRENDER [...]" (ANEP - CEIP, 2011, n.p.). Dado que el maestro comunitario, figura instituida por el PMC, representa un actor educativo fundamental en las escuelas APRENDER, los discursos pedagógicos que atraviesan su praxis tienen importantes efectos en el desarrollo de esta propuesta, más aún si se considera que APRENDER consiste, en gran medida, en una articulación de programas y proyectos preexistentes.

En el marco del programa APRENDER, las construcciones discursivas que conciben a los educandos desde la posibilidad y no desde la falta entran en tensión con otros sentidos que también lo atraviesan y constituyen, y que permiten percibir grandes continuidades con las significaciones consolidadas en el discurso de la reforma educativa de los años noventa. Esas construcciones de sentido refieren a la concepción de los sujetos de la educación como "sujetos carentes" (MARTINIS, 2006, 2013), a perspectivas que definen a los estudiantes que viven en contextos de pobreza a partir de sus carencias, de aquello que, según postulan, les falta para poder constituirse plenamente como sujetos de la acción educativa. Desde esta concepción, se establecen relaciones de determinación causal entre las condiciones de vida de ciertos sectores de la población y sus 
trayectorias educativas y características en tanto sujetos. De este modo, se postula que los niños que viven en contextos de pobreza no pueden aprender, se los ubica en un peldaño social y cultural inferior y se naturaliza su fracaso escolar.

Esta tensión se visualiza, por ejemplo, en el siguiente fragmento de un documento oficial, que hace referencia a la coordinación interinstitucional, en el marco del programa APRENDER, entre la ANEP y organismos vinculados a la protección social. Allí se plantea que:

El conjunto de programas y una articulación eficiente constituyen el "piso" de APRENDER, sin embargo parece que la educación de los más pobres (infelices en el vocablo artiguista) para que sean los más considerados (o privilegiados), exige aún más. lmpone un compromiso que trasciende lo que la escuela puede, y se proyecta sobre las condiciones de educabilidad (en los términos de Tedesco). (ANEP - CEIP, 2011, n.p.).

En este fragmento, ciertas características de los estudiantes, vinculadas a sus situaciones de vida, son concebidas como "condiciones de educabilidad" noción presente también en otros documentos- y se hace alusión a un autor clave en el desarrollo de dicho concepto. De este modo, se remite a una perspectiva que coloca bajo sospecha la calidad de educables de los niños de sectores populares, muchos de los cuales, según se evalúa, carecerían de estas condiciones.

En un sentido similar, en una publicación del programa se introduce la noción de "grupos de riesgo", los cuales, según se plantea, requerirían "[...] de atención diferencial y de propuestas que tuvieran presente la singularidad de los alumnos" (ANEP - CEIP, 2014, p. 20). El siguiente pasaje de dicho documento, en el que se describen las características que hacen que algunos niños sean incluidos dentro de esa categoría, expresa la noción de riesgo que se maneja:

El Programa trabajó para una definición sobre el significado de grupos de riesgo y ello implica pensar en diferentes características:

- alumnos que difieren de la mayoría en sus formas de actuar ya sea al relacionarse con sus pares o con los adultos, docentes o no docentes,

- alumnos que tienen formas no apropiadas de manejar su cuerpo quizás producto de una experiencia personal en donde la comunicación está mayormente situada en la reacción corporal,

- alumnos cuyo proceso escolar en relación a los aprendizajes está desfasado en cuando a los logros esperados en los tiempos previstos desde la Escuela [...],

- alumnos con entornos familiares vulnerables, que no presentan contención en alguna forma y que por alguna circunstancia no han establecido buena relación con el Centro Educativo desde lo social, académico o personal (ANEP - CEIP, 2014, p. 20). 
Se observa en este fragmento el proceso de clasificación que se desarrolla, no exclusivamente de las escuelas, sino también de los sujetos que asisten a ellas. Entre las características reseñadas, criterios que definen que algunos niños sean concebidos como miembros de los llamados "grupos de riesgo", solamente una tiene vinculación con sus desempeños académicos. Los otros tres factores aluden a aspectos disciplinarios y morales: de los niños, haciendo referencia a un manejo inadecuado de sus cuerpos y a formas de actuar que escapan a los parámetros de la normalidad, y de sus familias, asociando en este punto su carácter de "vulnerables" con la falta de contención. El énfasis en estos aspectos resulta indicativo de la importancia que se le otorga, especialmente en el trabajo con sujetos que viven en contextos de pobreza, a las prácticas de conducción y disciplinamiento.

Asimismo, la articulación de ese énfasis con la noción de riesgo remite a una lógica de la prevención, similar a la instalada en la década de los noventa. En dicho período, de acuerdo con Martinis (2013), se expandió en la sociedad uruguaya un creciente sentimiento de inseguridad, ligado a lo cual las poblaciones que vivían en situación de pobreza fueron construidas como poblaciones en riesgo y, a la vez, como peligrosas. En ese marco, la acción educativa fue concebida como una intervención que permitiría a los niños y adolescentes pobres pasar del territorio de la carencia al de la integración y, desde una lógica preventiva, evitar que estos desarrollaran "[...] aquellas conductas que por su situación social y cultural sería de esperar que asumieran" (MARTINIS, 2013, p. 162). Como sostiene este autor, se construyó entonces una cadena de equivalencias que articuló pobreza, fracaso educativo y delincuencia; si la educación fracasaba en su esfuerzo compensatorio, el sujeto carente mutaba en sujeto peligroso.

De este modo, las políticas educativas de los noventa, a partir de su vinculación con las ideas de riesgo y prevención, propusieron tecnologías de gobierno específicas para los niños que vivían en contextos de pobreza, privilegiaron la conducción de sus conductas y relegaron a un lugar subordinado a los aspectos vinculados al saber. Se observan en el programa APRENDER, creado en un contexto político y social sumamente diferente, profundas continuidades con relación a estas construcciones discursivas. Se identifica que, como plantea Martinis (2015), la producción de nuevos sentidos y la pretensión de los gobiernos progresistas de alterar ciertas lógicas hegemónicas en las décadas precedentes, se enfrentó a la dificultad de desmontar discursos que aún tienen un fuerte arraigo en el imaginario social, con su consecuente naturalización de la desigualdad y estigmatización de vastos sectores de la población.

En otro pasaje del mismo documento oficial, se afirma que, "Las escuelas A.PR.EN.D.E.R., integran en su matrícula a un alto porcentaje de alumnos que pertenecen a grupos de riesgo y habitualmente se puede visualizar que 
comparten algunos problemas producto de las situaciones anteriores, entre los más comunes está el rezago escolar" (ANEP - CEIP, 2014, p. 21). Se percibe aquí el nexo establecido entre aquellos factores que definen que ciertos estudiantes sean considerados "de riesgo" y sus contextos socioeconómicos, único criterio que define que una escuela sea APRENDER. A su vez, el empleo del término "producto" deja en evidencia la presunción de una relación causal entre dichos factores y los resultados educativos de los niños. Asimismo, parece no ser tenida en cuenta en este pasaje la única característica, entre aquellas enumeradas, referida al desempeño académico de los estudiantes, ya que ello tornaría tautológica la afirmación. De este modo, los procesos de enseñanza y de aprendizaje son colocados al final de una ecuación, en la cual los atributos de los estudiantes y sus familias son la causa y los resultados educativos la consecuencia. La responsabilización de los sujetos es aquí absoluta.

En otros fragmentos del mismo documento, esta posición se rechaza explícitamente y se asume una perspectiva contraria, por ejemplo al afirmar que la propuesta de APRENDER “[...] estuvo siempre pensando a la escuela como un espacio de lo posible, adjudicándole la capacidad de torcer destinos en relación a las trayectorias escolares que se presentaron durante muchos años como inevitables [...]" (ANEP - CEIP, 2014, p. 23). A su vez, en referencia a los resultados de una encuesta realizada a los docentes, se señala que:

[...] un porcentaje demasiado alto de maestros respondieron desde un lugar que hace pensar en "determinismo" que los docentes que trabajan en A.PR. EN.D.E.R. saben no es el sentir de la gran mayoría, pero no debiera ser el de ningún maestro porque un maestro con bajas expectativas también constituye un posible riesgo (ANEP - CEIP, 2014, p. 25).

En síntesis, con relación a las construcciones discursivas que atraviesan a ese programa, identificamos una tensión entre un posicionamiento que señala que todos los estudiantes pueden aprender, y que rechaza perspectivas deterministas, y ciertos sentidos que clasifican a poblaciones en función de sus carencias y anormalidades y responsabilizan a los sujetos por sus resultados educativos negativos.

Consideramos que para abordar ese dilema es preciso analizar todo el proceso de la política educativa, en el que son fundamentales tanto los documentos que la instituyen y regulan y los sentidos construidos desde espacios de gestión, como los modos en que los docentes producen la política en la práctica. Si bien en este artículo no nos abocamos al análisis de ese último punto, investigaciones 
previas (GEPPRED, 2018; MANCEBO; ALONSO, 2012) han arrojado importantes indicios de que esa tensión atraviesa también la praxis cotidiana de los maestros en las escuelas.

\section{CONSIDERACIONES FINALES}

A lo largo de este trabajo hemos discutido algunos sentidos construidos en torno a los sujetos de la educación cuando quienes ocupan esta posición son niños que viven en contextos de pobreza. Concretamente, nos aproximamos al programa APRENDER, una política educativa uruguaya con relación a la cual, orientadas por la perspectiva del Análisis Político del Discurso, procuramos identificar algunas regularidades en los discursos pedagógicos que la constituyen, ciertas fijaciones parciales de sentido, con sus tensiones y contradicciones, que dieran cuenta de modos particulares de pensar la escuela y, en especial, a los niños que asisten a ella. Esas regularidades se colocaron en relación con el devenir histórico del discurso educativo en Uruguay -que está inscrito, por supuesto, en un contexto mundial-, por medio de lo cual se pretendió contribuir al reconocimiento del carácter socio-histórico de los sentidos estabilizados. Entendemos que las interpretaciones que se ensayaron en este trabajo son, como todas, provisorias y abiertas, y que deberán ser rediscutidas, enriquecidas y reelaboradas en futuros trabajos de investigación y con relación a la nueva coyuntura política y social del país.

El corpus sobre el que se trabajó estuvo constituido por documentos oficiales referidos a la política de enseñanza primaria en Uruguay y a ese programa en particular, y por las transcripciones de entrevistas realizadas en el año 2019 a responsables y referentes de su gestión cotidiana. De este modo, se pretendió abarcar las construcciones de sentido que atravesaban el discurso oficial y el trabajo de actores con poder de decisión en el proceso de implementación de ese programa. Si bien no se abordó en este artículo, consideramos que es también fundamental analizar las posiciones construidas por los docentes en los procesos de producción de la política educativa desde el cotidiano escolar.

Identificamos en este trabajo tensiones en torno a las concepciones sobre los sujetos de la educación que constituyen al programa APRENDER, construcciones de sentido en las que se hibridan, en forma paradójica, elementos del discurso reformista de los años noventa con sentidos consolidados en el marco de los recientes gobiernos progresistas. Tanto en documentos macro de la política educativa, como en otros específicos de ese programa, conviven afirmaciones 
que señalan que todos los estudiantes pueden aprender con otras que colocan bajo sospecha esta posibilidad para el caso de algunos niños, en función de las carencias que, según se postula, estos portan.

Esos sentidos contradictorios están presentes, como dijimos, en los documentos institucionales analizados, mientras que en las entrevistas realizadas hubo referencias únicamente, si bien con ciertos matices, a una concepción que reivindica la decisión de educar partiendo de la posibilidad y no de la falta, de la convicción de que todos son capaces de establecer relaciones significativas con el conocimiento.

La característica central del programa APRENDER es su carácter focalizado, y su creación está estrechamente ligada al proceso, que se tornó sistemático, a mediados de los noventa, de clasificación de los centros educativos en función de sus contextos socioculturales. Ese programa se vincula a una idea que postula que es necesario construir propuestas diferenciadas para los niños que viven en contextos de pobreza, dicha idea se sustenta hoy en el concepto de inclusión educativa. Sobre la base de ese concepto, así como de las ideas de igualdad y equidad, y las distintas significaciones asociadas a estas nociones, se han desplegado históricamente políticas educativas con características diversas, que han oscilado entre una tendencia a la homogeneización, un reconocimiento de las diferencias y una naturalización de las desigualdades.

La discursividad educativa producida en Uruguay durante los quince años de gobierno progresista muestra procesos de hibridación discursiva (BORDOLI, 2018), a partir de los cuales ciertos elementos del discurso reformista de los noventa son afectados por construcciones discursivas recientes, que, por ejemplo, posicionan a la educación como un derecho universal. Con relación a los sujetos de la educación, se plantean disputas y se hibridan posiciones que colocan el foco en sus posibilidades de aprender y otras que los asocian a un conjunto de carencias. Los discursos que plantean como una verdad evidente que los niños que viven en condiciones de pobreza tienen, naturalmente, mayores dificultades para desarrollar aprendizajes o, directamente, que no pueden aprender, se instalaron con fuerza en el imaginario social y permearon también la discursividad educativa y las políticas desarrolladas en Uruguay por gobiernos de izquierda.

En marzo del 2020 terminó en el país un ciclo de 15 años de gobiernos progresistas y asumió el gobierno nacional una coalición de partidos políticos de centro-derecha. Ese cambio está, desde ya, dando lugar a nuevas luchas por hegemonizar el discurso educativo, las cuales, seguramente, transformarán los modos en que desde las políticas públicas se concibe a los estudiantes que viven en contextos de pobreza y, más en general, como se significan lo público, la educación, la igualdad y la justicia social. Consideramos que, en ese nuevo 
escenario social y político, la investigación en políticas educativas debe jugar un importante papel, aportando a la discusión pública sobre los sentidos que asumen las relaciones entre educación y desigualdad social, ya que de esas disputas dependerán las posibilidades de construir una escuela más igualitaria y justa.

\section{REFERENCIAS}

ANEP - CEIP. Documento 2o borrador para la discusión. Hacia un Modelo de Atención Prioritaria en Entornos con Dificultades Estructurales Relativas. Montevideo: ANEP-CEIP, 2010. a.

ANEP - CEIP. Acta N89, Resolución N³4. Montevideo, 2010b.

ANEP - CEIP. Acta Nº11, Resolución Nº9. Montevideo, 2011.

ANEP - CEIP. Haciendo memorias: Programa A.Pr.En.D.E.R. Montevideo: ANEP-CEIP, 2014.

ANEP - CEIP. Orientaciones de políticas educativas del Consejo de Educación Inicial y Primaria. Quinquenio 2016 - 2020. Montevideo: ANEPCEIP, 2016.

ANEP - CODICEN. Relevamiento de Contexto Sociocultural de las Escuelas de Educación Primaria 2010. Montevideo: ANEP-CODICEN, 2012.

BALL, Stephen John; MAGUIRE, Meg; BRAUN, Annette. Como as escolas fazem as políticas: atuação em escolas secundárias. Ponta Grossa: UEPG, 2016.

BARÁIBAR, Ximena. ¿Un puente hacia la nada?: Política Asistencial, Mundo del trabajo y Servicios Universales. Sociedade em Debate, [s. 1.], v. 22, n. 1, p. 104-140, 2016.

BORDOLI, Eloísa. Las políticas de inclusión educativa y los gobiernos progresistas. El caso uruguayo: algunos elementos analíticos para comprender los procesos políticos y educativos actuales. En: MONTIEN, María Cecilia; OLIVA, Dolores Leonor (Ed.). Educación, gobierno e instituciones en contextos diversos. 1a ed. San Luis: Laboratorio de Alternativas Educativas, 2012. p. 7-26. 
BORDOLI, Eloísa. La construcción de la relación pedagógica en la escuela uruguaya: sujetos, saber y gobierno de los niños. Montevideo: FHCEUdelaR, 2015. a.

BORDOLI, Eloísa. El Programa Maestros Comunitarios en el escenario discursivo posdictadura. Hilos que recuperan un discurso pedagógico. En: BORDOLI, Eloísa (Ed.). E1 Programa Maestros Comunitarios: Trazos, caminos y desafíos a la gramática escolar. Montevideo: FHCE-UdelaR, 2015. b. p. 91-111.

BORDOLI, Eloísa. El lugar de la teoría en la investigación educativa. Reflexiones en torno a la tensión teoría / empiria en el trabajo heurístico. Fermentario, [s. 1.], v. 1, n. 12, p. 105-118, 2018.

BUENFIL, Rosa Nidia. Análisis de discurso y educación. Documento DIE 26, Ciudad de México, 1993.

BUENFIL, Rosa Nidia. Apuntes sobre los usos de la teoría en la investigación social: consideraciones metodológicas en investigación social. Saarbrücken: EAE-LAP LAMBERT Academic Publishing GmbH \& Co. KG., 2011.

CASTEL, Robert. Las metamorfosis de la cuestión social: una crónica del salariado. Buenos Aires: Paidós, 1997.

DUHAU, Emilio. Política social, pobreza y focalización: reflexiones en torno al programa de educación, salud y alimentación. En: ZICCARDI, Alicia (Ed.). Pobreza, desigualdad social y ciudadanía: los límites de las políticas sociales en América Latina. Buenos Aires: CLACSO, 2001. p. 311-326.

FERRAÇO, Carlos Eduardo; CARVALHO, Janete Magalhães. Currículo: problematização entre práticas e políticas. Revista Teias, Rio de Janeiro, v. 13, n. 27, p. 3-7, 2012.

FLOUS, Clarisa. Una aproximación al análisis del discurso, en políticas y programas de inclusión educativa en la enseñanza media básica en el Uruguay (2005-2012). Políticas Educativas, Porto Alegre, v. 8, n. 2, p. 28-43, 2015.

GEPPRED. Informe Final: Proyecto "Mirándonos" [Mimeo]. Montevideo, 2018. 
LACLAU, Ernesto. La imposibilidad de lo social. En: LACLAU, Ernesto (Ed.). Nuevas reflexiones sobre la revolución de nuestro tiempo. Buenos Aires: Nueva Visión, 1993. p. 103-106.

LACLAU, Ernesto; MOUFFE, Chantal. Posmarxismo sin pedido de disculpas. En: LACLAU, Ernesto (Ed.). Nuevas reflexiones sobre la revolución de nuestro tiempo. Buenos Aires: Nueva Visión, 1993. p. 111-145.

MANCEBO, María Ester; ALONSO, Cecilia. Programa Aprender de Uruguay: Las visiones y opiniones de los maestros y directores. Montevideo: UNICEF, 2012.

MARTINIS, Pablo. Educación, pobreza e igualdad: del "niño carente" al "sujeto de la educación”. En: MARTINIS, Pablo; REDONDO, Patricia (Eds.). Igualdad y educación: escrituras entre (dos) orillas. Buenos Aires: Del Estante, 2006. p. $13-31$.

MARTINIS, Pablo. Educación, pobreza y seguridad en el Uruguay de la década de los noventa. Montevideo: CSIC-UdelaR, 2013.

MARTINIS, Pablo. Infancia y educación: pensar la relación educativa. Espacios en blanco. Serie indagaciones, [s. 1.], v. 25, n. 1, p. 105-126, 2015. Disponible en: http://www.scielo.org.ar/scielo.php?script=sci_arttext\&pid =S1515-94852015000100008. Acceso en: 20 jun. 2020.

URUGUAY. Ley General de Educación N 18.437. Uruguay, 2008.

\begin{abstract}
Camila Falkin
Doutoranda do Programa de Pós-Graduação em Educação da Universidade Federal do Rio Grande do Sul. Bolsista de doutorado do CNPq. Professora da Universidad de la República (Uruguai). E-mail: camifalkin@gmail.com

\section{Nalú Farenzena}

Doutora em Educação. Professora titular da Universidade Federal do Rio Grande do Sul, com atuação na Faculdade de Educação e no Programa de PósGraduação em Educação. E-mail: nalu.farenzena@gmail.com
\end{abstract}

